\title{
Research on Biodegradable Films and Their Comparison with Ordinary Films
}

\author{
YANG Yuqiu, WU Jingjing, LI Chunxiao, WANG Houwei and LIN Derong* \\ College of Food Science, Sichuan Agricultural University, Ya' an 625014, China \\ * Corresponding author
}

Keywords: Biodegradable film, Preservation, Package, Environmental protection.

\begin{abstract}
As a new type of film, degradable film is now widely used and can be used to package a variety of fruits, vegetables, etc., the purpose of which is to achieve better preservation and delay the storage period. With the development of modern biology, more and more people pay attention to degradable film. Degradable film not only has the function and characteristics of traditional plastic, but also it will revert to reductive form Ecological environment and return to nature in the life of life through the soil and water microbial action or by the role of sunlight in the ultraviolet light, in the natural environment, spliting degradation, so that it will not cause pollution to the environment. New-type fresh-keeping film of fruits and vegetables achieves the purpose of protecting the environment by using film made of degradable materials such as titanium dioxide, starch, soybean protein, chitosan and cellulose. However, now on the market many films do not have good preservation effect and can also destroy the flavor of the food. So this paper conducts a clear exposition of the development and preservation of biodegradable.
\end{abstract}

\section{Introduction}

The biodegradable plastic, a new kind of functional plastics, which refers to adding a certain amount of additives in the production process (such as starch, modified starch or other cellulose, photosensitizer, biological degradation agent, etc.), leads to decreasing stability and degrading plastic more easily in the natural environment ${ }^{[1]}$. Biodegradable plastics are made use of plant straw etc on the human body and environment friendly, which are different from three synthetic plastics. After they are abandoned with the action of biological environment, they can be resolved on their own. No matter for people or the environment harmless, the biodegradable plastic belongs to the green packaging. From the 1970s, researchers began to study the degradation of plastics abroad, in recent years, the research of biodegradable plastics and its production developed rapidly. Europe and the United States and Japan have developed a lot of products ${ }^{[2]}$. And some have been industrial production, widely used in agriculture ${ }^{[3]}$, packaging $^{[4]}$, health and other industries. British 
researchers developed degradable low-density polyethylene by adding ferrocene, light iron, benzophenone and other photosensitive materials in the polymer ${ }^{[5]}$. France, Germany, Israel and other countries have used this method to produce photodegradable polyethylene film, etc ${ }^{[6]}$. The use of photodegradable plastics is more, generally used in the manufacture of plastic bags, and less for food preservation in this area. China began to research photodegradable plastic from the 80s, also studying the biodegradable plastics in recent years ${ }^{[7]}$.

\section{The classification of the Biodegradable plastic}

Biodegradable plastics are generally divided into four major categories: photo degradable plastics, biodegradable plastics, photo biodegradable plastics, water degradable plastics. In the four kinds of degradable plastics, the development biodegradable plastics are getting better and better and become a new generation of hot spot.

\section{Study on Ordinary Thin Films}

Plastics in general are: polyethylene(PE), polyvinylidene chloride(PVDC), polyvinyl chloride (PVC), etc. PE is mainly used in food packaging, which sales on the market of household plastic wrap. The oxygen barrier property of PVDC is good, mainly used for cooking food, ham, and other products' packaging. But due to its difficult technology, this kind of material price is quite high, resulting that the plastic wrap is less on the market. PVC owns good transparency and it is not easily broken.

However, these films themselves have good chemical stability and are not easily destroyed. When they are abandoned, they will be in the environment for a long time, which are difficult to be decomposed, resulting in a large number of plastic waste in the environment accumulation to our planet, causing more serious pollution, such as white pollution, agriculture white cancer and so on $^{[8-13]}$. At the same time, oxidation usually causes a series of food deterioration reaction, seriously affecting the food shelf life. Currently, antioxidants are used to retard or inhibit the oxidation of food products. But these antioxidants will migrate into the food inside, not only affecting the taste of food, but also less than the role of preservation ${ }^{[14]}$. Therefore, developing an effective antibacterial and good biodegradable plastic wrap to prevent food contamination and reduce the oxidation rate to extend the shelf life are very important.

\section{The mechanism of biodegradable film}

For modified atmosphere packaging, the plastic wrap all needs to have a certain humidity and air permeability. It is mainly used to adjust the oxygen and water content and cut off the dust around product, aimed to prolong the shelf life of food and the purpose of freshness ${ }^{[15]}$. Mainly from the following aspects:

1.Adsorption. Here the object of the adsorption is mainly referred to the ethylene, because the ethylene has the role of ripening fruits and vegetables. Reducing the ethylene content in the packaging can effectively inhibit or slow down the process of fruit and vegetable maturation, the nutritional consumption and respiratory strength, thereby extending the shelf life. The adsorption of 
ethylene is mainly achieved by adding an ethylene absorbent to the wrap film.

2.Gas adjustment function. Gas is primarily involved the adjustment of $\mathrm{O}_{2}$ and $\mathrm{CO}_{2}$ concentrations. Maintaining the appropriate gas concentration in the packaging can effectively extend the shelf life of fruits and vegetables. As is known to all, $\mathrm{O}_{2}$ and $\mathrm{CO}_{2}$ are closely related to fruit and vegetable respiration. Reducing the concentration of $\mathrm{O}_{2}$ and increasing $\mathrm{CO}_{2}$ concentration can inhibit their own respiratory metabolism of fruits and vegetables and can reduce their own consumption, to extend the effect of fruit and vegetable preservation.

3.Anti-fog effect. The "fog" here is due to evaporation in the fruit and vegetable water vapor condensation on the surface of thin film formation. Packing in fog effect is not only the appearance of the fruits and vegetables, but also the quality of fruit and vegetable. Packaging too much water will breed bacteria, bringing about rotting fruits and vegetables. The addition of an antifogging agent to the wrap film can provide an effective improvement.

4.The antibacterial effect. There will always be some microbes on the surface of food, whose growth is easy to make food from spoiling. So adding certain antibacterial agent in plastic wrap to inhibit the growth of microbial food surface is better able to ensure food safety.

\section{The development trend of biodegradable film}

Starch is a good raw material for the preparation of biodegradable cling film. This is determined by the characteristics of starch. First, starch as glucose molecules polymerization and high molecular compound, is vulnerable to microbial decomposition, a membrane component which can make film degradation rate greatly increase. So it is applied to one of the important factors of the preparation of biodegradable film. Second, starch, cheap, as a kind of renewable resources, is one of the most widely used food raw materials. So the starch has a wider range of applications in the production of plastic packaging. For example, Yan et al used oxidative esterification starch as substrate, adding glycerin, sodium alginate, dehydrogenation sodium acetate to make antibacterial film, that is used in cake packaging ${ }^{[16]}$. Experiments show that the antibacterial film has higher security and can prolong the shelf life, being of great economic benefits.

Titanium dioxide is a good semiconductor and has good photocatalytic activity. So it can absorb the sun's ultraviolet rays, and then with the air in the oxygen and water to produce a very active free radicals. These free radicals can cause polymer molecular chain fracture and decomposition, and ultimately the plastic into carbon dioxide and water and other substances, to achieve the purpose of degradation. In addition, the titanium dioxide also has a strong stability, non-toxic and so on, so titanium dioxide as the preparation of photodegradable plastics preferred catalyst ${ }^{[17-20]}$. In recent years, photodegradable plastics prepared with titanium dioxide as photocatalyst have attracted much attention of researchers.

\section{Conclusions}

In this paper, we summarized the introduction of biodegradable film and its preservation performance compared to the ordinary film. Degradable films play an important role in this area of the fruit and vegetable preservation, not only because of its environmental protection, but also its preservation. Effectively extending the food storage period, but have better preservation effect, 
reducing the food damage rate. In the packaging materials, the amount of foreign degradable film is also great, which provides a larger potential export market for China's biodegradable film. At the same time, industrial developed countries limit the application of non-degradable plastics to be restricted or prohibited, which are for the development of China's biodegradable plastics industry to provide a market. Therefore, the market prospect of China is optimistic for degradable films. However, the degradation time of biodegradable cling film needs to be longer, so it is necessary to research more effective and rapid degradation materials.

\section{References}

[1] Kolybaba M,Tabil L G,Panigrahil S,et al. Biodegradable Polymers: Past, Present, and Future.In 2003 American Society of Agricultural Engineers annual Meeting, Fargo, North Dakata, USA, 2003.

[2] Xu Z H. Research and Development of Biodegradable Plastics in Japan [J]. Modern Chemical Industry, 1992, (05): 40-43

[3] Cui B Y,Zhang Z F,Kong Q. Application of Natural Biodegradable Materials in Agriculture [J]. Chinese agricultural science bullertin, 2013, (24): 5-10.

[4] Liang M,Wang Y,Song S X,et al. Application of Biodegradable Polymeric Materials in Food Packaging [J]. Plastics Industry, 2015, (10): 1-5 +18.

[5] Thomas, Noreen L,et al. Oxo-degradable plastics: degradation, environmental impact and recycling. Proceedings of the Institution of Civil Engineers: Waste and Resource Management; London165.3 (Aug 2012): 133-140.

[6] Su X. UK and Israel to develop new catalysts for PLA biodegradable plastics [N].China Packaging News, 2010-11-30 (002).

[7] Research and Application of Biodegradable Plastics in China [J]. New Materials for Chemical Engineering, 1995, (07): 35-44.

[8] Nampoothiri K M,Nair N R,John R P. An overview of the recent developments in polylactide(PLA) research [J]. Bioresource Technology, 2010, 101(22):8493-8501.

[9] Leja K, Lewandowice G. Polymer Biodegradation and Biodegradable Polymers-Review[J].Polish Journal of Environmental Studies, 2010, 19(2): 255-266.

[10] Kasirajan S,Ngouajio M. Polyethylene and biodegradable mulches for agricultural applications: a review [J]. Agronomy for Sustainable Development, 2012,32(2):501-529.

[11] Zheng Y, Yanful E K, Basai A S. A review of plastic waste biodegradation[J]. Critical Reviews in Biotechnology,2005,25(4): 243-250.

[12] Ammala A,Bateman S,Dean K,et al. An overview of degradable and biodegradable polyolefins[J]. Progress in Polymer Science,2011,36(8): 1015-1049.

[13] Shimao M. Biodegradation of plastics[J]. Current Opinion in Biotechnology,2001,12(3): 242-247.

[14] MastromatteO M,Mastromatteo M,Coonye A,et al. Advances in controlled release devices for food packaging applications[J]. Trends in Food Science \& Technology,2010,21(12): 591-598.

[15] Zhao S H. PCL / Preparation and Properties of PPC blend wrap film [D]. Hohhot:Inner Mongolia Agricultural University,2013,6: 2-2.

[16] Yan Q Q. Preparation and Properties of oxidized esterified starch-based antimicrobial food packaging film studies [D]. Taian: Shandong Agricultural University, 2012.

[17] Shang J,Chai M,Zhu Y F. Solid-phase photocatalytic degradation of polystyrene plastic with $\mathrm{TiO}_{2}$ as photocatalyst[J]. Journal of Solid State Chemistry, 2003,174(1): 104-110.

[18] Zan L,Fa W,Wang S. Novel photo degradable low-density polyethylene- $\mathrm{TiO}_{2}$ nanocomposite film[J]. Environmental Science \& Technology, 2006, 40(5): 1681-1685.

[19] Fa W J,Gong C Q,Tian L H,et al. Enhancement of photocatalytic degradation of poly(vinylchloride) with perchlorinated iron(2) phthalocyanine modified nano-TiO2 [J]. Journal of Applied Polymer Science,2011, 122(1): 1823-1828.

[20] $\mathrm{Li} \mathrm{S} \mathrm{Y,Xu} \mathrm{S} \mathrm{H,He} \mathrm{L} \mathrm{J,et} \mathrm{al.} \mathrm{Photocatalytic} \mathrm{degradation} \mathrm{of} \mathrm{polyethyrlene} \mathrm{plastic} \mathrm{with} \mathrm{polypyrrole} / \mathrm{TiO}_{2}$ nanocomposite as photocatalyst[J]. Polymer-Plastics Technology and Engineering, 2010, 49(4): 400-406. 